\title{
Moral Atmosphere and Masculine Norms in American College Football
}

\author{
Jesse Steinfeldt and Leslie A. Rutkowski \\ Indiana University \\ Thomas J. Orr \\ Northern State University \\ Matthew C. Steinfeldt \\ Fort Lewis College
}

\begin{abstract}
This study examined on-field antisocial sports behaviors among 274 American football players in the United States. Results indicated that moral atmosphere (i.e., teammate, coach influence) and conformity to masculine norms were significantly related to participants' moral behavior on the field (i.e., intimidate, risk injury, cheat, intentionally injure opponents). In other words, the perception that coaches and teammates condone on-field antisocial behaviors-in addition to conforming to societal expectations of traditional masculinity - is related to higher levels of antisocial behaviors on the football field. In addition, conformity to traditional masculine norms mediated the relationship between moral atmosphere and on-field aggressive sports behaviors, suggesting a relationship between social norms and moral atmosphere. Results of this interdisciplinary endeavor are interpreted and situated within the extant literature of both the fields of sport psychology and the psychological study of men and masculinity. Sport psychologists can use results to design interventions that incorporate moral atmosphere and conformity to masculine norms in an effort to decrease aggressive sports behaviors in the violent sport of football.
\end{abstract}

An abundance of researchers have investigated antisocial sports behaviors on the fields of play, often examining these behaviors within the context of moral functioning (e.g., Coulumb-Cabagno \& Rascle, 2006; Kavussanu, Roberts, \& Ntoumanis, 2002; Kavussanu, Stamp, Slade, \& Ring, 2009; Miller, Roberts, \& Ommundsen, 2005; Shields \& Bredemeier, 2007; Stephens \& Kavanagh, 2003; Stuart \& Ebbeck, 1995; Visek \&Watson, 2005). These authors have examined a variety of sports (e.g., soccer,

J. Steinfeldt and Rutkowski are with the Dept. of CEP, Indiana University, Bloomington, IN. Orr is with the Dept. of Sport Marketing and Administration, Northern State University, Aberdeen, SD. M. Steinfeldt is with the Dept. of Athletics, Fort Lewis College, Durango, CO. 
handball, basketball, hockey, volleyball) because the unique culture of each sport can provide athletes with differing messages about the appropriateness of antisocial sports behaviors (Shields \& Bredemeier, 2007). Because researchers have identified gender differences in the prevalence of antisocial sports behaviors (e.g., Kavussanu et al., 2009), it is important to consider the role of gender and corresponding gender ideologies operating within the context of each sport.

The sport of American football is believed to perpetuate ideologies of masculine superiority by valuing, encouraging, and sanctioning instrumental aggression (Gage, 2008; Messner, 1990, 1992; Pringle \& Markula, 2005). According to Kreager (2007), "Masculinized sports then become socially sanctioned stepping stones toward privilege and power-sites where coaches, peers, parents, and the media encourage masculine identities founded on physical aggression and domination" (p. 706). As a result, football provides myriad opportunities to explore on-field aggressive sport behaviors among the young men who participate in this influential institution of masculinity socialization (e.g., Foley, 2001; Steinfeldt, Steinfeldt, England \& Speight, 2009; Wong, Steinfeldt, LaFollette, \& Tsao, 2011). Subsequently, in this study we intended to examine the relationship between conformity to traditional masculine norms, moral atmosphere, and moral functioning in football. By doing so, we hoped to identify influences on college football players' on-field aggressive sports behaviors in this aggressive and often violent contact sport.

\section{Moral Atmosphere in Sport}

The moral atmosphere of a team-which may include the influence of coaches, teammates, and significant others (Kavussanu et al., 2002; Shields et al., 2007)-is considered to be an important factor in understanding how players choose whether to engage in aggressive sport behaviors on the fields of play (Shields \& Bredemeier, 2007). Aggressive sports behaviors have been defined as overt physical or verbal acts that can potentially physically or psychologically harm an opponent (Jamieson \& Orr, 2009). Although rules in sport exist to maintain the integrity of the game, there are often gray areas of interpretation (e.g., what acts might be considered strategic but are not in the spirit of the rules) that allow justifications for aggressive sports behaviors to occur on the field. According to Messner (1990),

Although most athletes will 'stretch' the rules as much as they can to gain an advantage over their opponents, most have respect, even a reverence, for the importance of rules as a code of conduct that places safe boundaries around their aggression and their relationships with others. Without the rules, there would be chaos-both physically and psychologically (p. 209).

Rest's $(1983,1984)$ model of moral action is a framework often used to examine aggressive sports behaviors on the fields of play (Shields \& Bredemeier, 2007). This model purports that in each moral action, there are four distinct yet interactive processes: (a) interpreting the situation; (b) forming a moral judgment about the appropriate thing to do; (c) deciding what one intends to do by selecting among competing values; and (d) engaging in the behavior. The second and third processes (i.e., judgment, intention) of Rest's model have been a primary focus of much of the moral functioning research in sport (Shields \& Bredemeier, 2007), but recent research has included the fourth process (i.e., behavior; e.g., Kavussanu et al., 2002). For example, research has shown that participation in high-contact sports (i.e., wrestling and judo) contributed to a greater likelihood 
that participants would aggress on the field (Bredemeier, Weiss, Shields, \& Cooper, 1986), and that men may engage in more antisocial on-field behaviors than women (Kavussanu et al., 2009). Thus, it is possible that the aggressive and violent nature of football (Gage, 2008; Messner, 1990) may produce ambiguity about what constitutes antisocial on-field behavior in this "masculinized" highcontact sport (e.g., Kreager, 2007).

\section{Conformity to Masculine Norms in Sport}

Social gender norms guide and constrain men's and women's understanding of how they are supposed to think, feel, and act in society (Mahalik et al., 2003). Specifically, masculine gender norms are socially constructed unwritten rules that convey strong messages about what it means to be a man (Addis \& Mahalik, 2003). According to conformity to masculine norms theory, men who feel greater pressure to conform to societal expectations of "what it means to be a man" are at greater risk for negative psychological functioning (Mahalik et al., 2003). Conformity to masculine norms theory has identified a subset of dominant norms of traditional masculinity that are prevalent in American society: (a) Winning; (b) Emotional Control; (c) Risk-Taking; (d) Violence; (e) Power Over Women; (f) Playboy; (g) Self-Reliance; (h) Primacy of Work; and (i) Heterosexual SelfPresentation. Research in the psychological study of men and masculinity has demonstrated that conforming to traditional masculine norms can exact a cost on men and on others in men's lives (e.g., Burn \& Ward, 2005; Liu \& Iwamoto, 2007; Locke \& Mahalik, 2005; Mahalik, Levi-Minzi, \& Walker, 2007; Mahalik, Talmadge, Locke, \& Scott, 2005; Parent \& Moradi, 2009). Even though sport is believed to serve as a primary influence on young men's construction of masculinity (Martin \& Harris, 2006; Messner, 1992; Wellard, 2002; Whannel, 2007), there has been a dearth of research into how masculine norms operate in sport. To this point, Wong, Steinfeldt, Speight, and Hickman (2010) reported that sport-related themes were found in only $1 \%$ of the studies in the last decade of scholarship in Psychology of Men \& Masculinity, the flagship journal for the Society for the Psychological Study of Men and Masculinity (Division 51 of the American Psychological Association [APA]).

Referred to as "part of the glue of masculine culture” (Messner, 1996, p. 77), sport is an influential environment wherein boys learn values and behaviors (e.g., competition, toughness, independence) that are considered to be valued aspects of masculinity within American society (Messner, 1992). Because many men participate in sport throughout their formative adolescent years (e.g., National Federation of State High School Associations [NFHS], 2010), the norms that operate within sport can significantly influence the process of masculinity development among athletes (Martin \& Harris, 2006). In addition, participation in different sports can have a unique impact on the process of masculinity socialization and subsequent development of norms that are enforced by team culture (Gage, 2008). Within the culture of the sport of football, players are required to use instrumental aggression to achieve the primary goals of dominating one's opponent to win (Messner, 1990). However, utilizing aggression and even violence should be considered a learned behavior in this context:

Despite the fact that few males truly enjoy hitting, and one has to be socialized into participating in much of the violence that is commonplace in sports, males 
appear to be predisposed to view aggression, within the rule-bound structure of sports, as legitimate, natural, and even 'safe' in a psychological sense (Messner, 1990, pp. 208-209).

Thus, the values, structure, and rules of football contribute to the development of a distinct set of norms which can elicit aggressive norm-congruent behavior among participants, and the norms of masculinity need to be empirically assessed to determine their role in this process.

\section{Current Study}

Researchers have demonstrated that higher levels of perceived moral atmosphere contribute to higher aspects of moral functioning in sport (e.g., Kavussanu et al., 2002; Kavussanu et al., 2009; Miller et al., 2005; Shields \& Bredemeier, 2007; Shields et al., 2007; Stephens \& Kavanagh, 2003). In addition, researchers in the psychological study of men and masculinity (e.g., Burn \& Ward, 2005; Liu \& Iwamoto, 2007; Locke \& Mahalik, 2005; Mahalik et al., 2007) have empirically linked conformity to masculine norms to a host of negative outcomes. However, no researchers to date have examined whether these norms influence on-field outcomes among athletes. Subsequently, the purpose of this study was to examine the influence of moral atmosphere and conformity to masculine norms on college football players' on-field moral functioning across the aspects of judgment, intention, and behavior. More simply stated, our research question is whether moral atmosphere is predictive of antisocial on-field behavior, both directly and as mediated by conformity to masculine norms among college football players.

To do so, we attempted to extend Kavussanu et al.'s (2002) framework of moral atmosphere and moral functioning into the domain of college football (see Figure 1 for the hypothesized conceptual model of this current study). Our first hypothesis was that lower levels of moral atmosphere (i.e., influence of teammates

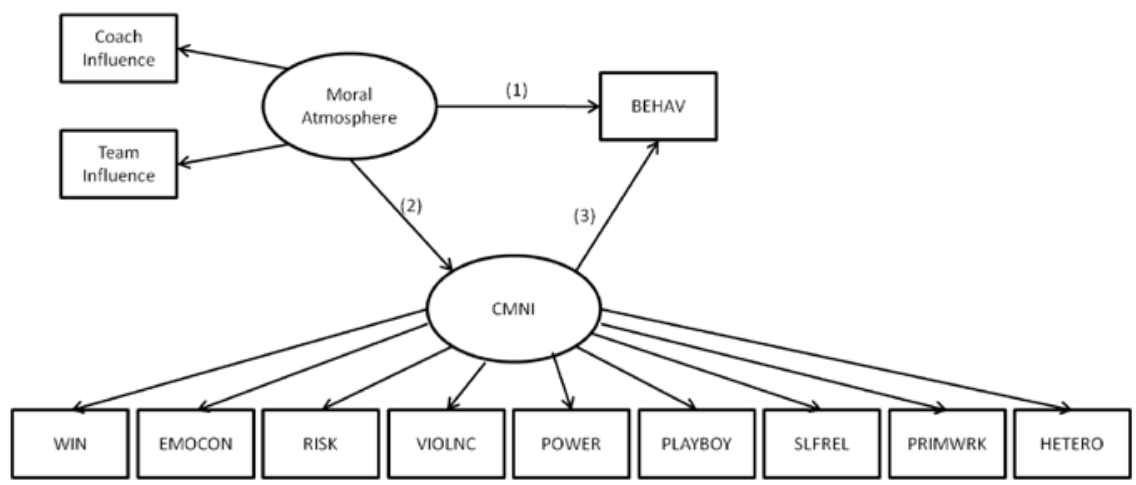

Note : WIN $=$ Winning; EMOCON $=$ Emotional Control; RISK $=$ Risk-Taking; VIOLNC $=$ Violence; PLAYBOY = Playboy; POWER = Power Over Women; SLFREL = Self-Reliance; PRIMWRK $=$ Primacy of Work; HETERO $=$ Heterosexual Self-Preservation; $\mathrm{CMNI}=$ Conformity to Masculine Norms; BEHAV = Moral Behavior.

Figure 1 - Theoretical model of moral functioning among college football players. 
and coaches; Path 1) and higher levels of conformity to masculine norms (Path 3 ) would predict lower aspects of moral functioning. That is, the lower a player perceives the moral atmosphere on his team to be and the more pressure he feels to conform to traditional masculine norms, the more likely he will be to demonstrate lower moral functioning on the field across the dimensions of judgment, intention, and behavior. Traditional masculine norms resonate with the tenets of hegemonic masculinity, which purport that organized sports perpetuate traditional ideologies of winning, dominance, aggression, and violence (Messner, 1992, 1996, 2002; Pringle $\&$ Markula, 2005). Thus, it is plausible that conformity to traditional masculine norms (e.g., Winning, Violence) may contribute to a player's propensity to engage in antisocial sports behaviors (e.g., cheating, risking injury to one's opponent) that are perceived as a necessary means to an end (i.e., winning the game). In addition, because past researchers have demonstrated that athletes at higher competitive levels perceived aggressive sports behaviors to be more acceptable (e.g., Visek \& Watson, 2005), we sampled football players from different divisions of play. Subsequently, we included Division of Play in our model (i.e., National Collegiate Athletic Association [NCAA] Division I, NCAA Division III), and hypothesized that NCAA Division I football players would report a greater likelihood of engaging in antisocial sports behaviors than NCAA Division III players.

Finally, we intended to examine if these traditional masculine norms indirectly influence the on-field moral functioning of college football players, in combination with the norms of moral atmosphere. Subsequently, our second hypothesis was that conformity to masculine norms would mediate the relationship between moral atmosphere and moral functioning (the product of Paths 2 and 3). According to Kavussanu et al. (2002), "many of the inappropriate actions we observe in the sport realm might be the result of certain social norms that become predominant in each team over time, thereby reinforcing unsportspersonlike behaviors" ( $\mathrm{p}$. 362). Subsequently, we hypothesized that peer pressure to conform to masculine norms may influence teammates' perceptions of overall moral atmosphere on the team, which may impact football players' decisions to engage in antisocial sports behaviors on the field.

\section{Method}

\section{Participants}

The participants in this study were 274 college football players who attended one of four universities in the Midwestern and Mideastern regions of the United States. Two of the colleges participated at the NCAA Division I (NCAA DI) level and two of the colleges participated at the NCAA Division III (NCAA DIII) level. The average age of the participants was $19.53(S D=1.33)$, and the sample consisted of 100 freshmen, 69 sophomores, 63 juniors, and 42 seniors. The sample self-identified their race as White (80\%), Black (15\%), Multiracial (2\%), or Other (3\%; e.g., Asian American, Hispanic). Fifty percent of participants reported playing an offensive position, $48 \%$ reported playing a defensive position, and $2 \%$ of participants were kickers or punters. Finally, because much attention has focused on the academic performance of student-athletes in revenue-producing sports (e.g., minimum 2.0 GPA required for athletic eligibility), it is noteworthy that the sample had an average overall GPA of $3.00(S D=0.48)$. 


\section{Procedures}

Research was conducted in compliance with Institutional Review Board approval from the first author's host institution. Athletic administrators and coaches were contacted, and they subsequently agreed to make players available to participate in the study. The survey packet was distributed to participants during a team meeting. Participants were assured of anonymity and were informed that all their data would be kept confidential and in a safe locked location. Participants took approximately 10-15 min to complete the survey packet. To ensure voluntary participation, participants were informed that if they did not want to participate in the study, they could write in their playbooks and turn in a blank survey packet at the end.

\section{Measures}

Conformity to Masculine Norms Inventory-46. The CMNI-46 (Parent \& Moradi, 2009) is a 46-item self-report instrument that uses a four-point Likert-type scale with possible responses ranging from 0 (strongly disagree) to 3 (strongly agree). The CMNI-46 is a psychometrically validated short form of the original 94-item CMNI (Mahalik et al., 2003). The purpose of the CMNI-46 is to assess men's conformity to various masculine norms that are widely endorsed in dominant American culture. The CMNI-46 has nine subscales: (a) Winning (e.g., "In general, I will do anything to win"); (b) Emotional Control (e.g., "I tend to keep my feelings to myself"); (c) Risk-Taking (e.g., "I enjoy taking risks"); (d) Violence (e.g., "Sometimes violent action is necessary"); (e) Power Over Women (e.g., "In general, I control the women in my life"); (f) Playboy (e.g., "If I could, I would frequently change sexual partners"); (g) Self-Reliance (e.g., "It bothers me when I have to ask for help"); (h) Primacy of Work (e.g., "My work is the most important part of my life"); and (i) Heterosexual Self-Presentation (e.g., "I would be furious if someone thought I was gay"). Some items are reverse-coded, and higher scores represent higher levels of conformity to masculine norms.

Parent and Moradi (2009) reported convergent validity evidence based on the CMNI-46 and its subscale factors being positively correlated with the theoretically corresponding scales of the original CMNI. Reliability for the CMNI-46 was demonstrated by the nine subscales producing internal consistency coefficients ranging from .77 (Primacy of Work) to .91 (Heterosexual Self-Presentation), and .88 for the overall CMNI-46 scale (Parent \& Moradi, 2009). Given the growing obsolescence of coefficient alpha as a measure of internal consistency, we adopted a more contemporary approach for the current study and used Guttman's (1945) $\lambda 2$, which is known to be a better lower bound to reliability, since $\alpha \leq \lambda 2 \leq \rho x$, (Sitsma, 2009) and may be a better bound than the greatest lower bound, $\rho \mathrm{glb}$, when sample sizes are relatively small (Ten Berge \& Sočan, 2004). To compare with the previously reported internal consistency estimates, we also report coefficient alpha. The internal consistency coefficients for the current study were as follows: Winning: $\alpha=.75$; $\lambda 2=.75$; Emotional Control: $\alpha=.86 ; \lambda 2=.86$; Risk-Taking: $\alpha=.80 ; \lambda 2=.81$; Violence: $\alpha=.63 ; \lambda 2=.66$; Power Over Women: $\alpha=.83 ; \lambda 2=.83$; Playboy: $\alpha=$ $.75 ; \lambda 2=.76$; Self-Reliance: $\alpha=.82 ; \lambda 2=.82$; Primacy of Work: $\alpha=.71 ; \lambda 2=$ .72 ; and Heterosexual Self-Presentation: $\alpha=.78 ; \lambda 2=.78$. It is important to note that although we report reliability coefficients for the individual subscales, each subscale enters the model as a single indicator, the collection of which serves as a measure of the conformity to male norms construct. 
Although internal consistency for the Violence subscale was low, the relationship between this indicator and the conformity to male norms construct, as measured by its standardized factor loading, was the strongest among all CMNI indicators (.576). Further, it had the lowest residual variance among all the CMNI indicators (.669). In addition, given the adequate fit of the confirmatory factor analytic model to the data for the CMNI indicators $\left(\chi_{18}^{2}=26.06, p=.10\right)$, there was sufficient evidence to support the inclusion of this indicator in subsequent models. Finally, despite a relatively low reliability for the violence subscale of violence, overall internal consistency for the total CMNI-46 scale was $\alpha=.85 ; \lambda 2=.86$.

Moral Functioning. Moral functioning was assessed by adapting a measure used by Kavussanu et al. (2002) to assess on-court moral functioning in their study of college basketball players. Through extensive consultation with academics, sport psychologists, and high school and college football coaches, the four scenarios in this measure were revised to be football-specific. The adapted scenarios used in this study are:

(a) Intimidate: During a critical football game, you have just knocked your opponent to the ground (after making a tackle or a block). To intimidate him when the referees are not looking, you push off of him to get yourself up off the ground.

(b) Risk Injuring Opponent: In the last minute of a critical football game, your team is leading by four points. However, the opposing team is driving toward your end zone. You get past the offensive lineman as you rush the quarterback, but you begin stumbling to the ground. The only way to get to the quarterback is to dive at his knees from the blind side. You have to decide whether to risk injuring the player to get the sack that potentially clinches the victory for your team.

(c) Cheating: It is the last minute of a critical football game, and your team is leading by two points. The other team has just completed its third pass in a row in their no huddle offense. They have crossed midfield and have momentum. To give your team rest and to make defensive substitutions, you stay on the ground after a play and fake an injury to stop the clock.

(d) Intentionally Injure Opponent: You are in a critical football game. After an interception (or during a punt/kick return), you are trailing far behind the play. You are running alongside the other team's best player, who is playing with bruised ribs. Because the player is merely jogging down the field, you can take him out of the game by hitting him with a ferocious blindside shot without being caught by the referee.

These scenarios were assessed across Rest's $(1983,1984)$ three aspects of moral functioning (i.e., judgment, intention, behavior). For each level of moral functioning, participants were asked after each scenario to rate how appropriate they thought the behavior was (judgment), whether they would engage in the behavior (intention), and whether they had ever engaged in the behavior (behavior). On a five-point Likert-type scale, with possible responses ranging from 1 (never appropriate for judgment and never for intention and behavior) to 5 (very often or always appropriate for judgment, always for intent, and very often for behavior), scores were summed across all four scenarios to provide a total score for each moral functioning level. The internal consistency for the Moral Judgment scale $(\alpha=.55 ; \lambda 2=.56)$ was lower than the 
coefficient alpha of .67 for this scale, reported by Kavussanu et al. (2002). The internal consistency coefficient for the Moral Intention scale $(\alpha=.57 ; \lambda 2=.58)$ was also lower than the value $(\alpha=.72)$ reported by Kavussanu et al. (2002), while the Moral Behavior scale $(\alpha=.73 ; \lambda 2=.74)$ was consistent with the coefficient alpha .70 reported by Kavussanu et al. (2002). Given the low internal consistency values for Moral Judgment and Moral Intention, we chose to omit these variables from subsequent models and instead focused only on Moral Behavior as our outcome variable.

Moral Atmosphere. We used the Moral Atmosphere measure from Kavussanu et al.'s (2002) study, which included the influence of both coaches and teammates. To assess Coach Influence across the four scenarios of moral functioning, participants were asked the degree to which they thought their coach would encourage the behavior in the scenario, if it was necessary to win. A five point Likert-type scale was used, with possible responses ranging from 1 (strongly discourage) to 5 (strongly encourage). To assess Teammate Influence across the four scenarios, participants were asked how many of their teammates they thought would engage in the behavior in each respective scenario. A five point Likert-type scale was used, with possible responses including 1 (none of the players) to 5 (everyone on the team). Scores were summed across all four scenarios to provide a total score for Coach Influence and for Teammate Influence. The internal consistency for both Coach Influence $(\alpha=.60 ; \lambda 2=.60)$ and Teammate Influence $(\alpha=.60 ; \lambda 2=.60)$ were lower than the coefficients alpha of .82 and .70 reported by Kavussanu et al. (2002) on the respective scales. In spite of these low values, well-fitting confirmatory factor models $\left(\chi_{2}^{2}=2.98, p=.22 ; \chi_{2}^{2}=4.79, p=.09\right)$ for Coach Influence and Teammate Influence, respectively) and statistically significant standardized factor loadings between each indicator and its construct provided evidence in favor of their inclusion for subsequent models.

For this model assessing the moral functioning and moral atmosphere variables, Kavussanu et al. (2002) used a Multitrait-Multimethod (MTMM) analysis to validate the measure of behavior and the two indicators (i.e., coach, teammate) of moral atmosphere across the four game reasoning scenarios. Utilizing confirmatory factor analysis and structural equation modeling, the results of their MTMM analysis demonstrated evidence of moderate convergent and discriminant validity. These findings provide some evidence that this instrument is appropriate to use as a valid measure for assessing moral atmosphere and functioning in sport.

\section{Item Parceling}

Based on our analytic strategy (i.e., structural equation modeling) discussed subsequently, an ideal model would incorporate individual indicators of each dimension and construct. For example, the Coach Influence dimension would be indicated by the four questionnaire items that tap that latent construct. Unfortunately, our sample size precluded this approach, which would have resulted in as many as 115 latent variables, including second order factor analyses for Moral Atmosphere and Conformity to Masculine Norms. As recommended by Little, Cunninham, Shahar and Widaman (2002), when interest is principally on the relations among latent variables, a parceling approach is justified as reasonable. As such, we chose an item parceling scheme that used adequately fitting confirmatory factor analyses to justify combining items into single indicators. Specifically, nonsignificant chisquare statistics and acceptably small normalized residuals (absolute value $<2.00$ ) were used as criteria for judging acceptable fit of the scale. 


\section{Analysis Strategy}

Based on suitable confirmatory factor analysis results, the following measures were comprised of their associated items: All nine CMNI-46 subscales, Coach Influence, Teammate Influence, and Moral Behavior. All item parcels used sums across the relevant items to create scores for the resultant indicators. To test the hypothesized model of relationships between Conformity to Masculine Norms, Moral Atmosphere, and Behavior, we used a structural equation modeling strategy with a maximum likelihood estimator and bootstrapped standard errors. To establish identification of the hypothesized model, we employed the recommended methods of McDonald and Ho (2002). To test the hypothesis that conformity to masculine norms mediates the relationship between moral atmosphere and behavior, we used methods, described in MacKinnon (1994), MacKinnon, Lockwood, and Williams (2004) and implemented in MPlus (Muthén \& Muthén, 2010). In particular, we followed MacKinnon (1994) to first examine the magnitude of the relationship between moral atmosphere and behavior while omitting CMNI from the model. We then tested for a significant relationship between moral atmosphere and CMNI in a model that excludes behavior. Finally, we tested whether the relationship between moral atmosphere and behavior was reduced by the inclusion of CMNI in the model and an indirect relationship between moral atmosphere and behavior that passed through CMNI. The full results are discussed subsequently; however, it is suffice to note that our findings provided justification for pursuing a statistical test of an indirect effect of moral atmosphere on behavior as mediated by conformity to masculine norms. To test for the significance of the indirect relationship, the standard errors for the product of the path coefficients of interest were generated using the bias corrected bootstrap method with 5000 bootstrap draws. Model fit was evaluated based on the chi-square test (nonsignificant), the root mean squared error of approximation (RMSEA < .05) with standard errors, and an examination of normalized residuals (absolute value $<2.0$ ). Further, we examined the possibility of model misspecification via the expected parameter change in combination with the modification index (MI) and the power of the MI test (Saris, Satorra, \& van der Veld, 2009). We set a minimum modification index value of 10.00 and a model misspecification value $(\delta)$ of .10. Large residuals and MIs led us to correlate theoretically sensible errors in observed variables. All models were fit with Mplus 6.0 (Muthén \& Muthén, 2010). Only the initial and final models are reported in the interest of space.

\section{Results}

Based on hypothesized differences based on division of play, we considered in our original model specification the inclusion of Division of play (NCAA DI or DIII) as a predictor of moral atmosphere, conformity to male norms, and antisocial sports behaviors. However, the inclusion of Division of Play did not significantly improve the fit of the model (as measured by a chi-square difference test $\left(\chi^{2}{ }_{9}=16.91, p=\right.$ $.05)$, and none of the coefficients associated with division were significant. As such, we did not include a Division of Play predictor in subsequent models. A table of descriptive statistics and the correlation matrix for the variables used in our models can be found in Table 1. Using traditional measures of fit, the original theoretical model (see Figure 1$)$ fit the data poorly $\left(\chi_{52}^{2}=174.14, p<.001, \mathrm{CFI}=.80, \mathrm{TLI}=.74\right.$, RMSEA $=.093,95 \%$ CI $[.078, .108])$. Based on an inspection of the residuals and 


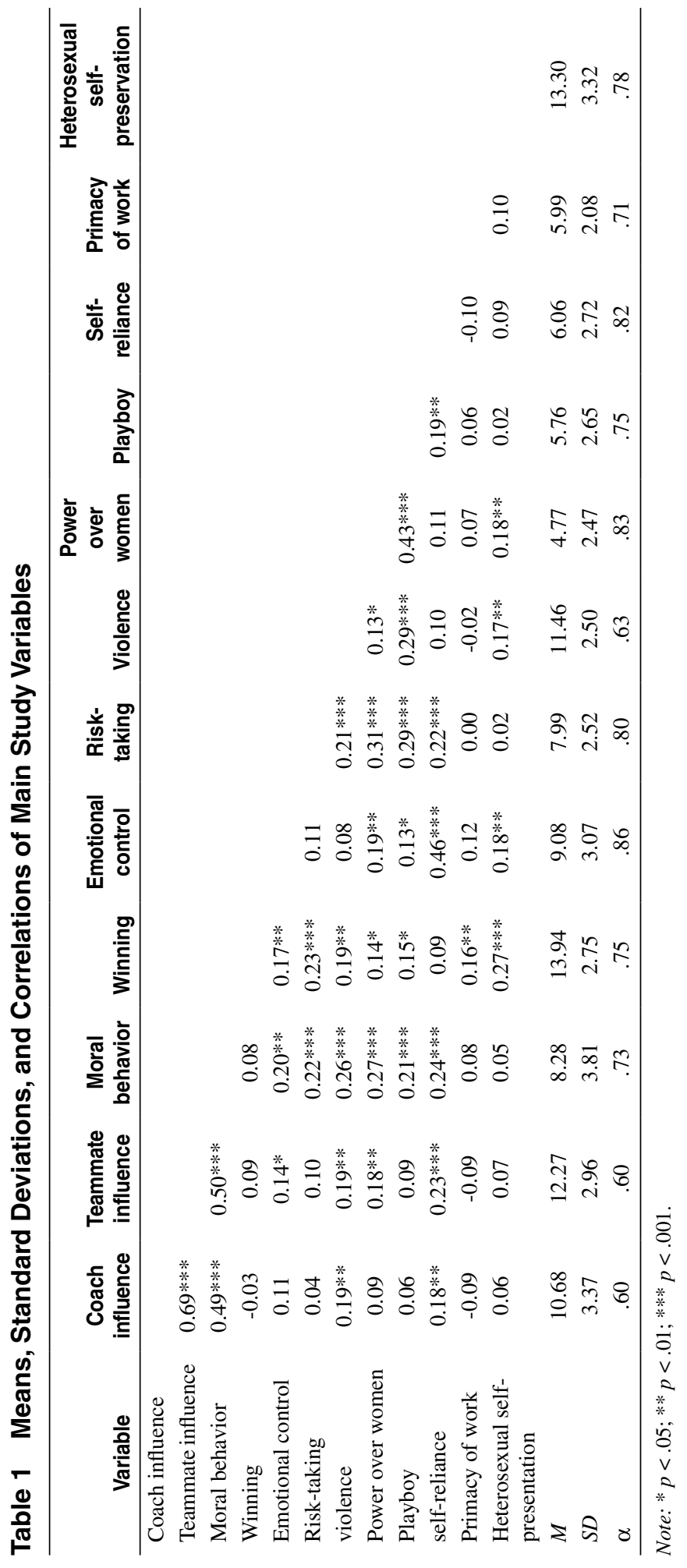


the model modifications suggested by an examination of the modification index, we proceeded with a model fitting process that correlated several theoretically reasonable observed variable errors (Bollen, 1989). The resultant model fit the data well ( $\chi_{34}^{2}=42.02, p=.16, \mathrm{CFI}=.99, \mathrm{TLI}=.97, \mathrm{RMSEA}=.029,95 \%$ CI $[.000, .056]$, all residuals $<2.0$ ). An investigation of model misspecification (Saris et al., 2009) indicated no theoretically tenable misspecifications.

The final model with standardized regression weights and the significance for each path is presented in Figure 2. A table of error variance and covariance based on correlated errors for the CMNI measurement model can be found in Table 2 . Given our interest in the relationships among the latent variables, we do not discuss the measurement model results. The paths from Moral Atmosphere to Behavior and Moral Atmosphere to CMNI were both positive and statistically significant, providing support for our first hypothesis. This suggests that a perception by players that coaches encourage poor behavior and that teammates would also engage in poor behavior has a positive effect on antisocial sports behavior and on the conformity by players to masculine norms. Furthermore, the path from CMNI to Moral Functioning was also positive and statistically significant, which suggests that conformity to masculine norms is significantly related to antisocial sports behavior on the football field.

To test our second hypothesis, we also pursued a mediation analysis in the vein of MacKinnon (1994). All results are standardized coefficients. Based on an examination of the magnitude of the relationship between moral atmosphere and behavior while omitting CMNI from the model, our results suggested a statistically significant and positive relationship between the two variables $\left(\beta_{\mathrm{MA} \text { to BEHAV }}=.591\right.$, $95 \%$ CI $[.516, .710])$. We then tested for a significant relationship between moral

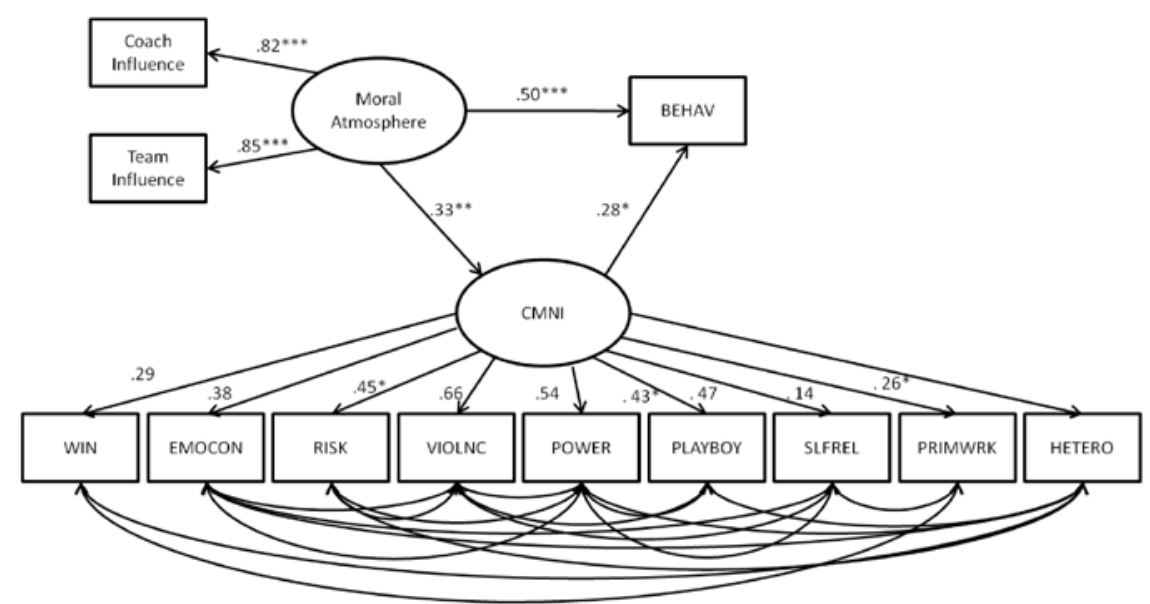

Note : WIN $=$ Winning; EMOCON $=$ Emotional Control; RISK $=$ Risk-Taking $;$ VIOLNC $=$ Violence; POWER = Power Over Women; PLAYBOY = Playboy; SLFREL = Self-Reliance; PRIMWRK = Primacy of Work; HETERO = Heterosexual Self-Preservation; CMNI = Conformity to Masculine Norms; BEHAV $=$ Moral Behavior.

${ }^{*} p<.05 ; * *<.01 ; * * * p<.001$.

Figure 2 - Final model of moral functioning among college football players $(N=274)$. 


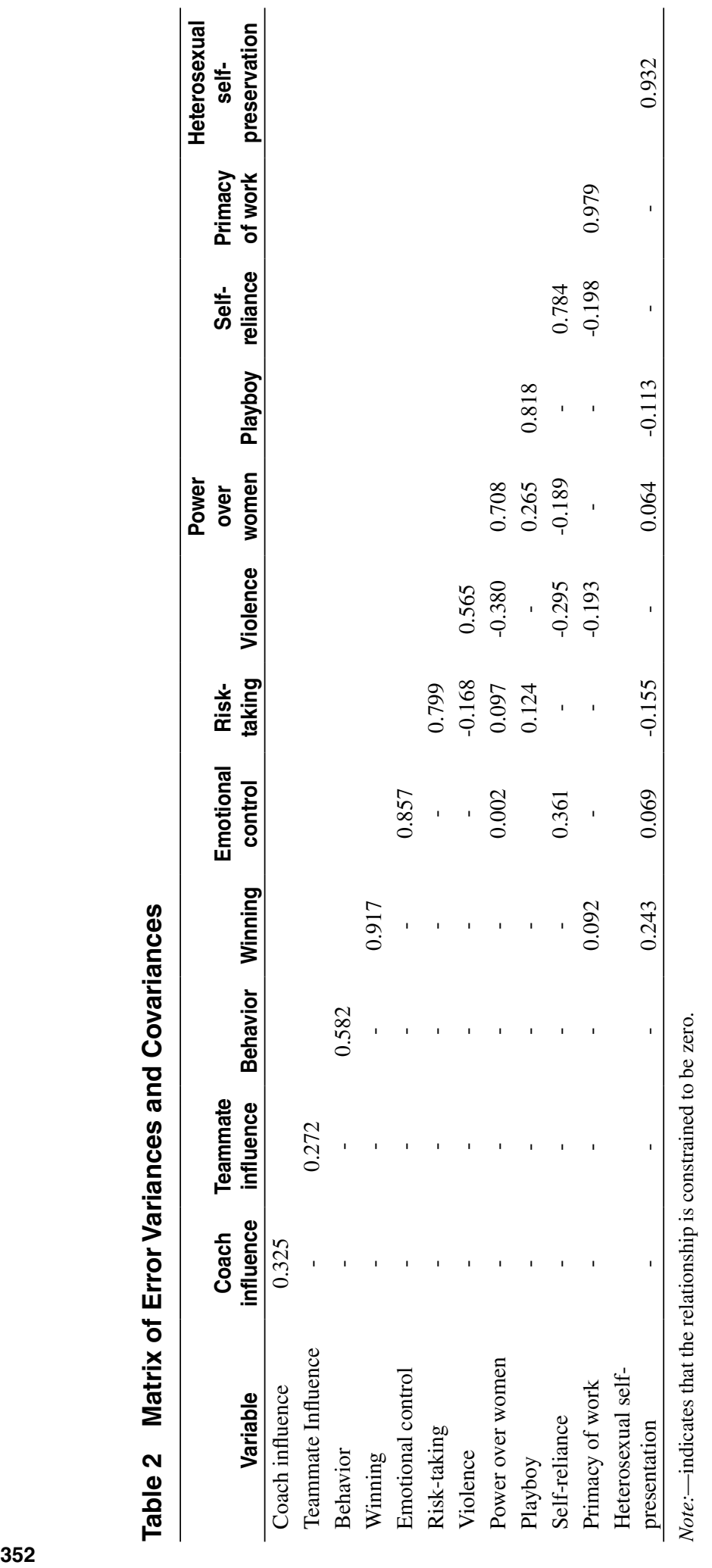


atmosphere and CMNI in a model that excludes behavior $\left(\beta_{\mathrm{MA} \text { to CMNI }}=.14495 \% \mathrm{CI}\right.$ $[.210, .510])$. Finally, we tested whether the relationship between moral atmosphere and behavior was reduced by the inclusion of CMNI in the model and an indirect relationship between moral atmosphere and behavior that passed through CMNI (from .591 to .499). Finally, the indirect effect between Moral Atmosphere and Behavior, as mediated by CMNI, was positive and statistically significant $\left(\beta_{\text {indirect }}=\right.$ $.9295 \%$ CI [.040, .143], $\mathrm{p}<.01)$. In support of our second hypothesis, this finding provides evidence of partial mediation (Judd \& Kenny, 1981a, 1981b) whereby the positive relationship between moral atmosphere and behavior is partially mediated by conformity to masculine norms.

\section{Discussion}

The results provided support for the study's first hypothesis that lower levels of moral atmosphere (i.e., coach influence, teammate influence) and higher conformity to traditional masculine norms would be significantly related to on-field moral behavior. In other words the perception that coaches and teammates condone onfield antisocial behaviors-in addition to pressure to conform to norms of traditional masculinity-is strongly and positively related to higher levels of antisocial sports behaviors on the field. This finding is consistent with past research in sport psychology on the relationship between moral atmosphere and moral functioning in sport (Guivernau \& Duda, 2002; Kavussanu et al., 2002; Miller et al., 2005; Kavussanu \& Spray, 2006; Stephens, 2000; Stephens \& Kavanagh, 2003). The results speak to the influence of perceived moral atmosphere in football, a context wherein aggression and violence are neither accidents nor violations of the rules, but are instead intended and desired dimensions of the game (Messner, 1990). In addition, because of the belief that, "Sports-related violence is an all-too-common occurrence at all levels of sport...Usually the public discounts or ignores all but the most extreme violence because it is boys being boys" (Fields, Collins, \& Comstock, 2007, p. 360 ), the results of this study stress the importance of examining conformity to masculine norms. Doing so can assure that the "boys being boys" explanation can be empirically assessed and deconstructed. Appropriate interventions can subsequently be created to minimize sports related violence.

In addition to directly impacting on-field moral functioning, conforming to traditional masculine norms also served as a mediator between: (a) the influence of coaches and teammates who create and enforce moral atmosphere norms that operate on the football team; and (b) antisocial sports behavior on the field of play. In support of our second hypothesis, the significant indirect relationship between moral atmosphere and moral behavior, as mediated by conformity to masculine norms, highlights the masculinity socialization processes operating within this sport. Pressure to "be a man" by conforming to traditional masculine norms may impact a player's decision-making process as it relates to engaging in on-field antisocial behaviors (e.g., intimidation, intentionally injuring one's opponent) that reflect an exaggerated misperception-a perception that can influence other teammates-of the aggressive and violent nature of the game of football (e.g., Gage, 2008; Messner, 1990). In sum, examining the relationship between masculine norms, moral atmosphere, and aggressive sports behaviors can help us better understand lessons that young men learn while participating in this influential institution of masculinity socialization (e.g., Steinfeldt et al., 2011). 


\section{Limitations}

This study has a number of limitations to note. First, the design is cross-sectional. The experience of football players at these four universities may not generalize to the experience of other college football players in different geographic regions or from different backgrounds. Although we hypothesized that participants from different divisions of play (i.e., NCAA DI, NCAA DIII) would report differing levels of antisocial sports behaviors, the results of this study did not support this hypothesis. Future researchers should individually examine participants from different divisions to determine whether differences in norms and culture exist based on competitive levels of play. In addition, there were methodological limitations to this study. The psychometric properties of some of the measures used in this study represent a limitation to our results. Low reliability scores on Moral Judgment and Moral Intention scales caused us to drop these scales from the model, which may suggest that studying moral variables in the context of American football is more nuanced and complex than can be captured with a survey instrument. Future researchers could address these limitations by using behavioral observations to triangulate self-report results. For example, instead of vignettes future researchers could use video clips to provide players with visual representations of intended aggressive sports behaviors that were described in the scenarios. Similarly, the reliability scores on some of the other scales (i.e., Violence, Teammate Influence, Coach Influence) were lower than the values reported in previous studies, which has the potential to distort the relationship between the indicators and their latent constructs. These indicators were maintained for inclusion in the full model based on well-fitting confirmatory factor analytic models and statistically significant factor loadings between the indicators and their underlying construct. Because football players may conceptualize violence as a normal and even instrumental part of the game, the lower reliability estimate on the Violence subscale of the CMNI-46 suggests that more nuanced measurements of violence might be necessary to assess participants in the unique context of football. In sum, results-and subsequent conclusions based on these results-should be interpreted in light of these limitations.

\section{Future Research and Implications for Sport Psychologists}

Although sport is considered an influential context wherein traditional conceptions of masculinity develop and thrive (e.g., Messner, 1996; Steinfeldt et al., 2009), little research to date has addressed the relationship between masculinity socialization and on-field outcomes. However, an emerging body of research has examined the relationship between masculinity and off-field outcomes within football, highlighted by a special section entitled Recent Research on College Football Players that recently appeared in the APA journal, Psychology of Men \& Masculinity. The studies within this section addressed a variety of areas of masculinity (e.g., gender role conflict, conformity to masculine norms, drive for muscularity) that were found to be significantly related to a number of psychosocial outcomes (e.g., self-esteem, body image, life satisfaction, stigma toward help-seeking) among men who play football (Steinfeldt, Gilchrist, Halterman, Gomory, \& Steinfeldt, 2011; Steinfeldt, Wong, Hagen, Hoag, \& Steinfeldt, 2011; Wong et al., 2011). All of these manuscripts also provided recommendations for utilizing interventions related to masculinity socialization for psychologists who engage in clinical work with college football players. 
Subsequently, sport psychologists can use the results of this study to design interventions that aim to educate football players, coaches, and administrators about the importance of social norms and moral atmosphere in relation to on-field outcomes (i.e., antisocial sports behaviors). Specifically, sport psychologists can help football coaches understand their role in creating a moral atmosphere that can shape the moral behavior of their players, in teaching them to play the game 'the right way.' Because the results suggest that teammates also wield an influence over moral atmosphere, sport psychologists can help players understand their role in creating and enforcing a climate of ethical play on the field among their teammates. Because social norms can effectively legitimize even antisocial behavior (e.g., Kavussanu et al., 2002), empirically designed psychological interventions may be necessary to increase the likelihood of decreasing and even eliminating aggressive sport behaviors within the aggressive and often violent context of football (e.g., Gage, 2008; Messner, 1990).

In addition, recent research has suggested that college football coaches not only wield significant influence on the masculinity development of their players, but coaches also value the role sport psychologists can play in this process (Steinfeldt et al., 2011). Based on their interviews with college football coaches, the authors concluded,

A greater understanding of the role of these influential sources of masculinity socialization in sports can help psychologists provide training to coaches-perhaps, as one coach mentioned, to develop a masculinity playbook for coaches to use-so that coaches can provide optimal growth experiences to the young men whose lives they so greatly influence (2011, p. 257).

In sum, sport psychologists can work with coaches to implement interventions that help football players better understand the societal pressures that influence conformity to traditional masculine norms, based on their internalization of masculinity messages they receive throughout their experiences in football. Facilitating conversations about "what it means to be a man" with football players can help sport psychologists assist these men in making more conscious decisions about the costs and benefits of conforming to societal norms of traditional masculinity. These conversations can help players better understand how the sporting environment conveys both adaptive (e.g., be responsible, be accountable) and potentially maladaptive (e.g., be tough, be stoic, don't ask for help or show weakness) messages about masculinity. Adding the content area of masculinity socialization into our field's collective clinical repertoire can enhance sport psychologists' clinical competency as it relates to working with male student-athletes. Interventions related to masculinity socialization can be effective in helping football players use the positive life lessons sport teaches them so that these young men can be successful men both on and off the field.

\section{References}

Addis, M.E., \& Mahalik, J.R. (2003). Men, masculinity, and the contexts of help-seeking. The American Psychologist, 58, 5-14. PubMed doi:10.1037/0003-066X.58.1.5

Bollen, K. (1989). Structural equations with latent variables. New York: Wiley.

Bredemeier, B.J., \& Shields, D.L. (1986). Game reasoning and interactional morality. The Journal of Genetic Psychology, 147, 257-275. doi:10.1080/00221325.1986.9914499

Bredemeier, B.J.L., Weiss, M.R., Shields, D.L., \& Cooper, B. (1986). The relationship of sport involvement with children's moral reasoning and aggression tendencies. Journal of Sport Psychology, 8, 304-318. 
Burn, S.M., \& Ward, A.Z. (2005). Men's conformity to traditional masculinity and relationship satisfaction. Psychology of Men \& Masculinity, 6, 254-263. doi:10.1037/15249220.6.4.254

Cialdini, R.B. (2007). Descriptive social norms as underappreciated sources of social control. Psychometrika, 72, 263-268. doi:10.1007/s11336-006-1560-6

Cialdini, R.B., \& Trost, M.R. (1999). Social influence: Social norms, conformity, and compliance. In D. Gilbert, S. Fiske, \& G. Lindzy (Eds.), The handbook of social psychology (Vol. 2, pp. 151-192). Boston: McGraw-Hill.

Coulumb-Cabagno, G., \& Rascle, O. (2006). Team sports players' observed aggression as a function of gender, competitive level, and sport type. Journal of Applied Sport Psychology, 36, 1980-2000. doi:10.1111/j.0021-9029.2006.00090.x

Fields, S.K., Collins, C.L., \& Comstock, R.D. (2007). Conflict on the courts: An interdisciplinary review of sports related violence literature. Trauma, Violence \& Abuse, 8, 359-369. PubMed doi:10.1177/1524838007307293

Foley, D.E. (2001). The great American football ritual: Reproducing race, class, and gender inequality. In A. Yiannakis \& M.J. Melnick (Eds.), Contemporary issues in sociology of sport. Champaign, IL: Human Kinetics.

Gage, E.A. (2008). Gender attitudes and sexual behavior. Violence Against Women, 14, 1014-1032. PubMed doi:10.1177/1077801208321987

Gould, D., Collins, K., Lauer, L., \& Chung, Y. (2007). Coaching life skills through football: A study of award winning high school football coaches. Journal of Applied Sport Psychology, 19, 16-37. doi:10.1080/10413200601113786

Guivernau, M., \& Duda, J. (2002). Moral atmosphere and athletic aggressive tendencies in young soccer players. Journal of Moral Education, 31, 67-85. doi:10.1080/03057240120111445

Guttman, L. (1945). A basis for analyzing test-retest reliability. Psychometrika, 10, 255-282. PubMed doi:10.1007/BF02288892

Jamieson, L.J., \& Orr, T.J. (2009). Sport and violence: A critical examination of sport violence in the world. London: Elsevier.

Kavussanu, M., Roberts, G.C., \& Ntoumanis, N. (2002). Contextual influenes on moral functioning of college basketball players. The Sport Psychologist, 16, 347-367.

Kavussanu, M., \& Spray, C.M. (2006). Contextual influences on moral functioning of male youth footballers. The Sport Psychologist, 20, 1-23.

Kavussanu, M., Stamp, R., Slade, G., \& Ring, C. (2009). Observed prosocial and antisocial behaviors in male and female soccer players. Journal of Applied Sport Psychology, 21, S62-S76. doi:10.1080/10413200802624292

Kreager, D.A. (2007). Unnecessary roughness: School sports, peer networks, and male adolescent violence. American Sociological Review, 72, 705-724. doi: $10.1177 / 000312240707200503$

Little, T.D., Cunningham, W.A., Shahar, G., \& Widaman, K.F. (2002). To parcel or not to parcel: Exploring the question, weighing the merits. Structural Equation Modeling, 9, 151-173. doi:10.1207/S15328007SEM0902_1

Liu, W.M., \& Iwamoto, D.K. (2007). Conformity to masculine norms, Asian values, coping strategies, peer group influences, and substance use among Asian American men. Psychology of Men \& Masculinity, 8, 25-39. doi:10.1037/1524-9220.8.1.25

Locke, B.D., \& Mahalik, J.R. (2005). Examining masculinity norms, problem drinking, and athletic involvement as predictors of sexual aggression in college men. Journal of Counseling Psychology, 52, 279-283. doi:10.1037/0022-0167.52.3.279

MacKinnon, D.P. (1994). Analysis of mediating variables in prevention intervention studies. In A. Cazares and L. A. Beatty (Eds.), Scientific methods for prevention intervention research (pp.127-153). Washington, DC: NIDA Research Monograph 139, DHHS Pub. 94-3631. 
MacKinnon, D.P., Lockwood, C.M., \& Williams, J. (2004). Confidence limits for the indirect effect: Distribution of the product and resampling methods. Multivariate Behavioral Research, 39, 99-128. PubMed doi:10.1207/s15327906mbr3901_4

Mahalik, J.R., Levi-Minzi, M., \& Walker, G. (2007). Masculinity and health behaviors in Australian men. Psychology of Men \& Masculinity, 8, 240-249. doi:10.1037/15249220.8.4.240

Mahalik, J.R., Locke, B.D., Ludlow, L.H., Diemer, M.A., Scott, R.P.J., Gottfried, M., \& Freitas, G. (2003). Development of the Conformity to Masculine Norms Inventory. Psychology of Men \& Masculinity, 4, 3-25. doi:10.1037/1524-9220.4.1.3

Mahalik, J.R., Talmadge, W.T., Locke, B.D., \& Scott, R.P.J. (2005). Using the Conformity to Masculine Norms Inventory to work with men in a clinical setting. Journal of Clinical Psychology, 61, 661-674. PubMed doi:10.1002/jclp.20101

Martin, B.E., \& Harris, F.H. (2006). Examining productive conceptions of masculinities: Lessons learned from academically driven African American male student-athletes. Journal of Men's Studies, 14, 359-378. doi:10.3149/jms.1403.359

McDonald, R.P., \& Ho, M. (2002). Principles and practice in reporting structural equation analyses. Psychological Methods, 7, 64-82. PubMed doi:10.1037/1082-989X.7.1.64

Messner, M.A. (1990). When bodies are weapons: Masculinity and violence in sport. International Review for the Sociology of Sport, 25, 203-220. doi:10.1177/101269029002500303

Messner, M.A. (1992). Power at play: Sports and the problem of masculinity. Boston: Beacon Press.

Messner, M.A. (1996). Masculinities and athletic careers. In E.N. Chow, D. Wilkinson, \& M. Baca Zinn (Eds.), Race, class and gender: Common bonds, different voices (pp. 70-86). Thousand Oaks, CA: Sage.

Messner, M.A. (2002). Taking the field: Men, women and sports. Minneapolis: University of Minnesota Press.

Miller, B.W., Roberts, G.C., \& Ommundsen, Y. (2005). Effects of perceived motivational climate on moral functioning, team moral atmosphere perceptions, and the legitimacy of intentionally injurious acts among competitive youth football players. Psychology of Sport and Exercise, 6, 461-477. doi:10.1016/j.psychsport.2004.04.003

Muthén, L. K., \& Muthén, B. O. (1998-2010). Mplus (Version 6). Los Angeles, CA: Muthén \& Muthén.

National Federation of State High School Associations. (NFHS; 2010). 2009-10 high school athletics participation survey. Retrieved online from http://www.nfhs.org/content.asp $\mathrm{x}$ ?id=3282\&linkidentifier=id\&itemid=3282

Parent, M.C., \& Moradi, B. (2009). Confirmatory factor analysis of the Conformity to Masculine Norms Inventory and development of the Conformity to Masculine Norms Inventory-46. Psychology of Men \& Masculinity, 10, 175-189. doi:10.1037/a0015481

Pringle, R., \& Markula, P. (2005). No Pain Is Sane After All: A Foucauldian Analysis of Masculinities and Men's Experiences in Rugby. Sociology of Sport Journal, 22, 472-497.

Rest, J.R. (1983). Morality. In P. Mussen (Ed.), Manual of child psychology. J. Flavell \& E. Markman (Eds.), Vol. 3: Cognitive development (4 ${ }^{\text {th }}$ ed.; pp. 356-629). New York: Wiley.

Rest, (1984). The major components of morality. In W. Kurtines \& J. Gewirtz (Eds.), Morality, moral behavior, and moral development (pp. 356-429). New York: Wiley.

Saris, W., Satorra, A., \& van der Veld, W. (2009). Testing structural equation models or detection of misspecification? Structural Equation Modeling, 16, 561-582. doi:10.1080/10705510903203433

Schwartz, S.H. (1977). Normative influences on altruism. In L. Berkowitz (Ed.), Advances in experimental social psychology (Vol. 10, pp. 221-279). New York: Academic Press.

Shields, D.L., \& Bredemeier, B.L. (2007). Advances in sport morality research. In G. Tenenbaum \& R. Eklund (Eds.), Handbook of Sport Psychology (3rd ed., pp. 662-684). Hoboken, NJ: Wiley \& Sons, Inc. 
Sitsma, K. (2009). On the use, the misuse, and the very limited usefulness of Cronbach's alpha. Psychometrika, 74, 107-120. PubMed doi:10.1007/s11336-008-9101-0

Steinfeldt, J.A., Foltz, B.D., Mungro, J., Speight, Q.L., Wong, Y.J., \& Blumberg, J. (2011). Masculinity socialization in sports: Influence of college football coaches. Psychology of Men \& Masculinity, 12, 247-259. doi:10.1037/a0020170

Steinfeldt, J.A., Gilchrist, G.A., Halterman, A.W., Gomory, A., \& Steinfeldt, M.C. (2011). Drive for muscularity and conformity to masculine norms among college football players. Psychology of Men \& Masculinity, 12, 324-336. doi:10.1037/a0024839

Steinfeldt, J.A., Steinfeldt, M.C., England, B., \& Speight, Q.L. (2009). Gender role conflict and help-seeking stigma among college football players. Psychology of Men \& Masculinity, 10, 261-272. doi:10.1037/a0017223

Steinfeldt, J.A., Wong, Y.J., Hagan, A.R., Hoag, J.M., \& Steinfeldt, M.C. (2011). A contextual examination of Gender Role Conflict among college football players. Psychology of Men \& Masculinity, 12, 312-323.

Stephens, D. (2000). Predictors of likelihood to aggress in youth soccer: An examination of coed and all-girls teams. Journal of Sport Behavior, 23, 311-325.

Stephens, D.E., \& Kavanagh, B. (2003). Aggression in Canadian youth ice hockey: The role of moral atmosphere. International Sports Journal, 7, 109-119.

Stuart, M.E., \& Ebbeck, V. (1995). The influence of perceived social approval and moral development in youth sport. Pediatric Exercise Journal, 7, 270-280.

Ten Berge, J., \& Sočan, G. (2004). The greatest lower bound to the reliability of a test and the hypothesis of unidimensionality. Psychometrika, 69, 613-625. doi:10.1007/ BF02289858

Thompson, E.H., \& Pleck, J.H. (1986). The structure of male norms. The American Behavioral Scientist, 29, 531-543. doi:10.1177/000276486029005003

Visek, A., \& Watson, J. (2005). Ice hockey players' legitimacy of aggression and professionalization of attitudes. The Sport Psychologist, 19, 178-192.

Wellard, I. (2002). Men, sport, body performance and the maintenance of exclusive masculinity. Leisure Studies, 21, 235-248. doi:10.1080/0261436022000030641

Whannel, G. (2007). Mediating masculinities: The production of media representations in sport. In C. Carmichael Atkinson (Ed.), Sport and gendered identities: Masculinities, femininities, and sexualities (pp. 7-21). London: Routledge.

Wong, Y.J., Steinfeldt, J.A., LaFollette, J.R., \& Tsao, S-C. (2011). Men's tears: Football players' evaluations of crying behavior. Psychology of Men \& Masculinity, 12, 297-311. doi:10.1037/a0020576

Wong, Y.J., Steinfeldt, J.A., Speight, Q.L., \& Hickman, S.J. (2010). Content analysis of the Psychology of Men \& Masculinity (2000 to 2008). Psychology of Men \& Masculinity, 11, 170-182. doi:10.1037/a0019133 